\title{
ASYMMETRIC BILATERAL VARIATIONS IN THE MUSCULOCUTANEOUS AND MEDIAN NERVES WITH HIGH BRANCHING OF BRACHIAL ARTERY
}

\begin{abstract}
Vandana Tomar, Surbhi Wadhwa
Department of Anatomy, University College of Medical Sciences, Delhi 110095, India

Summary: Brachial Plexus is formed by the union of the anterior rami of cervical 5, 6, 7, 8 and thoracic 1 nerves. These nerves unite and divide to form the key nerves innervating the upper limb. Variations in the course of these nerves are clinically important to anesthetists, neurologists and orthopedicians. We report bilateral variations in the arterial and neural structures in the upper limb of a 65 year old cadaver. The muscles of the arm on one side were innervated by the median nerve with absence of musculocutaneous. While on the other side the musculocutaneous nerve contributed to the formation of the median nerve. There was a presence of high bifurcation of brachial artery on both sides. Knowledge of such variations in the innervations of muscles and the arterial supply of the limbs are important to remember before performing any reconstructive procedures or interventions on the limb.
\end{abstract}

Key words: Musculocutaneous nerve; Median nerve; Branching pattern; Brachial artery; Cubital fossa

\section{Introduction}

The upper limb is innervated by the brachial plexus which is formed by union of the anterior rami of cervical 5, 6, 7, 8 and thoracic 1 nerves. These nerves unite and divide to ultimately form the key terminal nerves supplying the flexor and extensor compartments of the upper limb. As a result of multiple union and divisions of these nerves variations are common in the brachial plexus. Musculocutaneous nerve, a branch of the lateral cord of brachial plexus, after its formation supplies the coracobrachialis and then pierces it. The nerve then supplies the remaining flexor muscles of the arm namely brachialis and biceps brachii to continue as the lateral cutaneous nerve of the forearm. The median nerve is formed by contributions from both the lateral and medial cord. It usually does not supply the muscles of the arm, though however it gives vascular branches to the brachial artery and articular branches to the elbow joint (11). Any variation in the course and distribution of the nerves and arteries has a bearing on various surgical procedures and reconstructions involving the $\operatorname{limb}(2,5,18,27)$.

\section{Case Report}

During routine cadaveric dissection of the axilla and upper arm in a 65 year old male cadaver in Department of Anatomy, University College of Medical Sciences, Delhi, an anomalous musculocutaneous nerve, variant distribution of median nerve and a proximal division of the brachial artery were detected on both sides. On the left side the musculocutaneous nerve arose normally from the lateral cord of brachial plexus. Thereafter, it pierced the coracobrachialis muscle without supplying it (Figure 1). It ran downwards in the plane between the biceps brachii and brachialis. The lateral division of lateral cord and medial division of medial cord together formed the median nerve. At a distance of $9.5 \mathrm{~cm}$ after the formation of median nerve, a branch from the median nerve coursed upwards to supply the coracobrachialis. At the level of insertion of coracobrachialis a communication existed between the median and musculocutaneous nerve. The musculocutaneous nerve continued to supply the biceps brachii and brachialis and form the sensory lateral cutaneous nerve of the forearm. Also at the same level the brachial artery gave a muscular branch to supply the muscles of the arm which crossed over the communication between the median nerve to musculocutaneous nerve. The brachial artery bifurcated proximally in the arm, into a radial and an ulnar artery (Figure 1,2$)$. The ulnar artery ran posterior to the median nerve in the distal part of the arm, crossed it and was the most medial structure in the cubital fossa. The structures in the cubital fossa from medial to lateral side were ulnar artery, median nerve, radial artery, tendon of biceps brachii and the radial nerve (Figure 3 ). The lateral cutaneous nerve of the forearm, a continuation of the musculocutaneous nerve appeared between the biceps brachii and brachialis and was seen anterior to the flexor muscles. The ulnar artery ran superficial to flexors of the forearm and after giving the usual branches crossed over the flexor retinaculum to form the superficial palmar arch and contribute to the formation of deep palmar arch. 


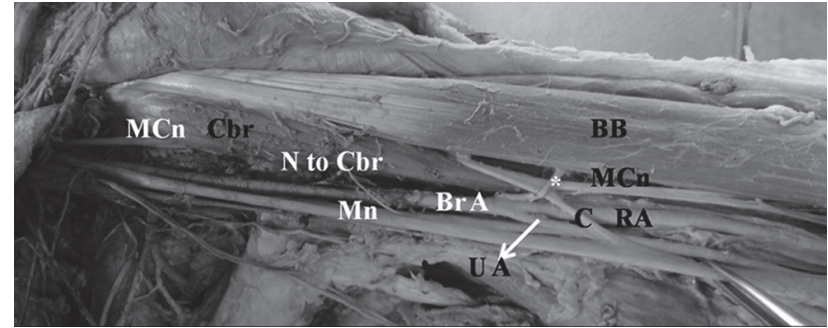

Fig. 1: Dissected specimen of left axilla and arm showing musculocutaneous nerve (Mcn) piercing the coracobrachialis (Cbr). Median nerve (Mn) supplying the $\mathrm{Cbr}$ ( $\mathrm{N}$ to $\mathrm{Cbr}$ ) and communicating $(\mathrm{C})$ with $\mathrm{Mcn}$ in the middle of the arm. Proximal division of brachial artery (Br A) into a radial (RA) and ulnar (UA) artery. A muscular branch (*) of brachial artery coursing over the communication. $\mathrm{BB}-\mathrm{Biceps}$ brachii

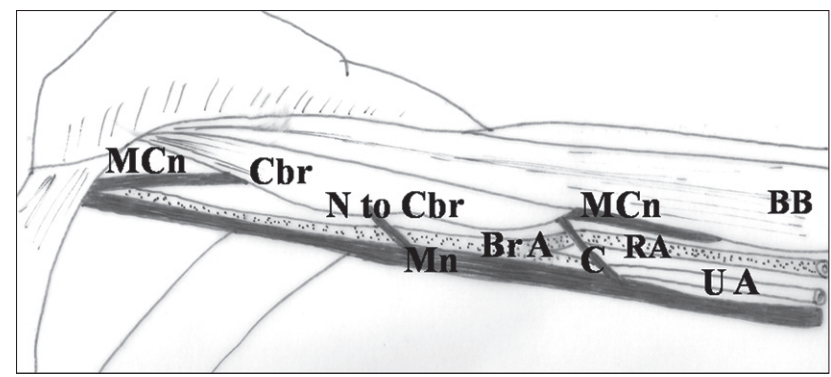

Fig. 2: Schematic diagram showing the coracobrachialis (Cbr) being supplied by median nerve (Mn). Mn sends a communication $(\mathrm{C})$ to musculocutaneous nerve $(\mathrm{MCn})$ in the middle of arm. Proximal division of brachial artery $(\mathrm{Br} \mathrm{A})$ into a radial (RA) and ulnar (UA) artery. BB - Biceps brachii

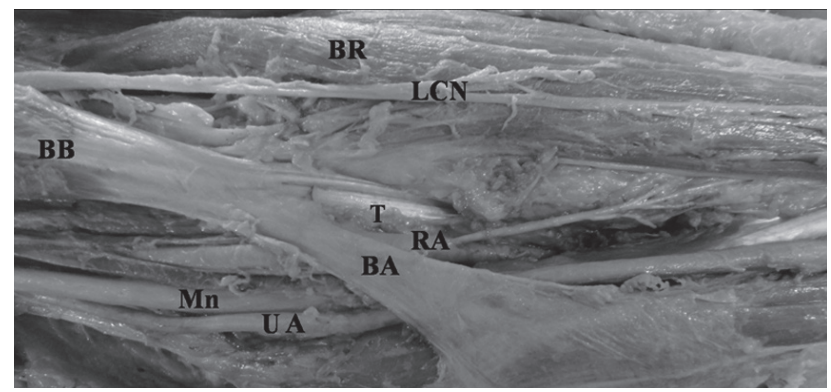

Fig. 3: Specimen of the left cubital fossa depicting the abnormally placed structures from medial to lateral side were ulnar artery (UA), median nerve $(\mathrm{Mn})$, radial artery (RA), and tendon of biceps brachii (T). BB - Biceps brachii; BA - bicipital aponeurosis; LCN - Lateral cutaneous nerve of Forearm; B - Brachioradialis

In the right axilla and arm, the musculocutaneous nerve was absent. The coracobrachialis was directly supplied by a nerve from the lateral cord (Figure 4, 5). The lateral and the medial cord joined to form the median nerve which gave a branch (B2) that immediately bifurcated to give a branch to biceps brachii and another branch, which was joined by a branch (B3) to form the lateral cutaneous nerve of the forearm (Figure 4,5). The brachial artery divided at the level of the elbow superficial to the median nerve into a normal radial and ulnar artery (Figure 4, 5). The rest of the arm did not show any abnormality.

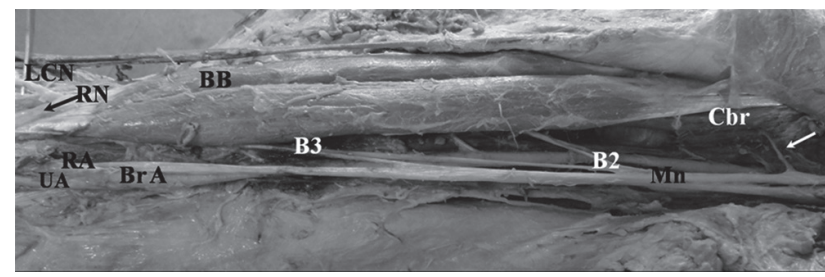

Fig. 4: Photograph of the right axilla and arm showing the coracobrachialis (Cbr) being supplied by lateral cord (arrow). A branch (B2) from the median nerve that immediately bifurcated to give a branch to biceps brachii and brachialis and another branch which was joined by a branch (B3) to form the lateral cutaneous nerve of the forearm (LCN). The brachial artery ( $\mathrm{Br} \mathrm{A}$ ) divided superficially in the cubital fossa into a normal radial (RA) and ulnar artery (UA). The radial nerve (RN, arrow marked) was seen lateral to the bicipital tendon

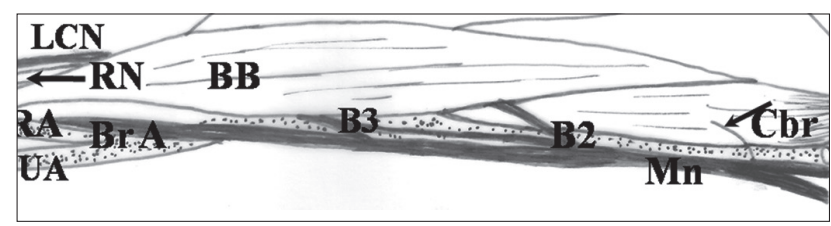

Fig. 5: Line diagram depicting the right axilla and arm. Note the absence of musculocutaneous nerve. The coracobrachialis (Cbr) is being supplied by lateral cord of brachial plexus (arrow). The remaining muscles of the arm - biceps brachii (BB) and brachialis (not shown) being supplied by branches from median nerve (B2). A branch B3 joins a branch from the muscular branch to form the lateral cutaneous nerve of the forearm (LCN). The brachial artery (Br A) divided superficially in the cubital fossa into a normal radial (RA) and ulnar artery (UA). Note the radial nerve (RN) lying lateral to the bicipital tendon 


\section{Discussion}

In the present case we found a bilateral variation in the innervation of the muscles of the arm and proximal bifurcation of brachial artery. On the left side there was a musculocutaneous nerve which did not supply the coracobrachialis, the latter being supplied directly by the median nerve, a distal communication between the median nerve and the musculocutaneous nerve, a proximally dividing superficial brachial artery, and hence an abnormal arrangement of structures at the cubital fossa on the left side. On the opposite side, the musculocutaneous nerve was absent and the median nerve supplied the muscles of the arm and cutaneous supply to the lateral side of the forearm. The brachial artery although normal became superficial in the cubital fossa and was the medial most structure here. It divided into its terminal branches in the elbow, however, more proximally than usual.

Iwata (1960) reported that during development brachial plexus arises as a single radicular cone of axons of spinal nerves. These nerves reorganize to form ventral and dorsal divisions. The median nerve forms from the ventral divisions and the musculocutaneous nerve later branches out from it (1). The nerve fibers of cervical 5 and 6 which were to form the musculocutaneous nerve may sometimes take a variant path (20). These may course through the lateral cord, thereafter travel in the median nerve and then rejoin the musculocutaneous nerve. This hitch hiking of the cervical 5, 6 fibers during development may be explained by the effect of neurobitaxis (24). In some cases, musculocutaneous nerve maybe absent. This is postulated to be a remnant of the primitive nerve supply of the arm $(9,13)$. In lower vertebrates like the amphibians, reptiles and birds there is only one nerve equivalent to the median nerve supplying the musculature of the thoracic limb (15). Parchand and Patil (2012) believe that absent musculocutaneous nerve as seen in this case on the right side is an example of recapitulation theory which states ontogeny recapitulates phylogeny.

Surprisingly in the present case the formation and distribution pattern of the two key nerves of the upper limb, median and musculocutaneous nerve varied on both sides. In the cadaver, on the left side the median nerve supplied the coracobrachialis and communication existed between the musculocutaneous and median nerve. The median nerve thus in this case is formed from three roots - the usual contributions from the medial and lateral cord and an additional root from the musculocutaneous nerve. This root may be inadvertently damaged during surgeries or trauma to the arm resulting in median nerve palsy (26). Any nerve with an unusual course or distribution is more likely to get injured (16). The right side upper arm in the present case was innervated by the median nerve with absent musculocutaneous nerve. Median nerve palsy in such cases would also result in inability of the patient to flex his elbow and loss of sensation on the lateral side of the forearm resulting in diagnostic errors (17). Knowledge of the anatomy of the brachial plexus and the relevant surrounding structures is of utmost importance to ensure safe and successful regional anesthesia of the limb. A variant distribution and branching of the musculocutaneous nerve is important to remember during regional anesthesia of the arm.

The unusual innervations of coracobrachialis by the median nerve or directly from the lateral cord are important to remember while using the muscle as flaps in deformities of infraclavicular and axillary areas and in reconstruction surgeries of the breast (14). Coracobrachialis is also advocated as an alternative to gracilis (muscle of the lower limb) for use in restoration of facial expressions in long standing cases of facial paralysis. One of the reasons for these muscles to be chosen was their reliable neurovascular pattern (25). Variations in the innervations of coracobrachialis are also important to surgeons performing surgeries on the coracoids process (6).

During development of the arteries of the upper limb, Rodriguez-Baeza et al. (1995) proposed the formation of axial artery from the 7th cervical intersegmental artery. This axial artery is the precursor of axillary, brachial and anterior interosseous artery. Concurrently, a superficial brachial artery originates from the axial artery by sprouting of several trunks. This superficial brachial artery gives rise to superficial antebrachial artery, and a laterally situated branch that represents the superficial part of the definitive radial artery. The superficial antebrachial artery gives rise to the definitive ulnar artery. The final arterial pattern of the vessels which forms depends on the dominance/regression of various arterial trunks and anastomosis formed between the superficial and deep vessels (21). In the present case the superficial brachial artery seen is a developmental anomaly.

High division of brachial artery into radial and ulnar has been reported also by Celik et al. (4). In one of their cases the artery bifurcated near the formation of median nerve and in the other just below the origin of profunda brachii artery. In the present case on one side the brachial artery bifurcated in the middle of the arm into radial and ulnar artery and on the other at the elbow but superficially. Thus the terminal branches were superficially placed at the elbow in both cases. The ulnar artery which originates high up in arm and remains superficially placed in the forearm has been named superficial brachioulnar artery by Rodriguez-Niedenfuhr et al. (1999, 2001). It has an incidence of $4.2 \%$ in adult population $(22,23)$. Kian et al. (2011) reported a high bifurcation of the brachial artery in about $12.3 \%$ of patients. The presence of this anomaly, if unrecognized, is associated with higher occurrence of dialysis access failure (12). The knowledge of these variations also is essential while performing percutaneous brachial artery catheterization in order to avoid complications (7). The possibility of abnormally placed structures in the cubital fossa must be kept in mind by vascular, plastic surgeons and radiologists. Venipuncture is usually done to collect 
blood from this site and vessels if abnormally placed as in the present case may get punctured. The cubital fossa is also used for arterial cannulation and is an area for anesthetic access to nerves of the forearm (8). Basche et al. prefer a transbrachial approach to a femoral artery catheterization for various radiological interventions because of its lower complication rate (3). Hence knowledge of the placement of structures at the cubital fossa becomes important.

\section{Conclusion}

This case is unique in its presentation as a bilateral asymmetric variation in the nerves and the brachial artery. Neurological lesions of median nerve in a case such as described in the present study, with absent musculocutaneous nerve, may present with confounding signs and symptoms to the unsuspecting clinician. The knowledge of variations in the nerve supply of coracobrachialis, communications between median and musculocutaneous nerve, variably placed structures in the cubital fossa as reported in this case assume considerable importance while performing orthopedic and vascular access procedures upon the upper limb.

\section{References}

1. Arey LB. Developmental anatomy: a textbook and laboratory manual of embryology. 7th ed. Philadelphia: W.B.Saunders, 1966: 429-435, 500-516.

2. Baliyan R, Mehta V, Arora J, Nayyar AK, Suri RK, Rath G. Unilateral interchordal neural communication coexistent with variant branching pattern of posterior cord of brachial plexus. Acta Medica (Hradec Kralove) 2011; 54(3): 131-4

3. Basche S, Eger C, Aschenbach R. The brachial artery as approach for catheter interventions- indications, results, complications. Vasa 2004; 33(4): 235-8.

4. Celik HH, Sargon MF, Konan A, Kural E. High brachial artery bifurcation: a report of 2 cases. Bull Assosc Anat (Nancy) 1996; 80 (250): 13-4.

5. Das S, Paul S. Anomalous branching pattern of brachial plexus. Int. J. Morphol 2005; 23(4): 289-92.

6. Das S, Maatoq Sulaiman I, Hussan F, Haji Suhaimi F, Latiff AA, Othman F. An unusual case of additional branches of median nerve innervating the corachobrachialis muscle and its clinical implications. Clin Ter 2009; 160(1): 25-7.

7. Deligonul L, Gabliani G, Kern MJ, Vandormael M. Percutaneous brachial catheterization: the hidden hazard of high brachial artery bifurcation. Cathet Cardiovasc Diagn 1988; 14(1): 44-5.
8. Ellis H, Feldman S, Harrup-Griffiths W. The clinical anatomy of the antecubital fossa. Br J Hosp Med (Lond) 2010; 71(1): 4-5.

9. Guerri-Guttenberg RA, Ingolotti M. Classifying Musculocutaneous Nerve Variations. Clin Anat 2009; 22: 671-83.

10. Iwata H. Studies on the development of the brachial plexus in Japanese embryo. Rep Dept Anat Mie Prefect Univ Sch Med 1960; 13: 129-44.

11. Johnson D. Pectoral Girdle and Upper limb. In: Standring S, ed. Gray's anatomy. The anatomical basis of clinical practice. London: Churchill Livingstone Elsevier, 2008: 791-837.

12. Kian K, Shapiro JA, Salman L, et al. High brachial artery bifurcation: clinical considerations and practical implications for an arteriovenous access. Semin Dial 2012; 25(2): 244-7.

13. Koizumi M, Sakai T. The nerve supply to coracobrachialis in apes. J Anat 1995; 186: 395-403.

14. Kopuz C, Icten N, Yildirim M. A rare accessory coracobrachialis muscle: a review of literature. Surg Radiol Anat. 2003; 24(6): 406-10.

15. Kosugi K, Mortia T, Yamashita H. Branching pattern of the musculocutaneous nerve. 1. Cases possessing normal biceps brachii. Jikeakai Med J 1986; 33: 63-71.

16. Kumar H, Das S, Rath G. An anatomical insight into the third head of biceps brachii muscle. Brastil Lek Listy. 2008; 109(2): 76-8.

17. Meals RA, Calkins ER. Anomalous innervation of the upper extremity. In: Gelberman R, ed. Operative Nerve Repair and Reconstruction. Philadelphia: Lippincot, 1991: 197-211.

18. Pacholoczak R, Klimek-Piotrowska W, Walocha JA. Absence of musculocutaneous nerve associated with supernumerary heads of biceps brachii: a case report. Surg Radiol Anat 2011; 33(6): 551-4.

19. Parchand MP, Patil ST. Absence of musculocutaneous nerve with variations in course and distribution of the median nerve. Anat Sci Int. 2012; DOI-10.1007 /s12565-011-0126-6.

20. Prasada Rao PV, Chaudhary SC. Communication of the musculocutaneous nerve with the median nerve. East Afr Med J 2000; 7: 498-503.

21. Rodriguez-Baeza A, Nebot J, Ferreira B, et al. An anatomical study and ontogenetic explanation of 23 cases with variations in the main pattern of the human brachio-antebrachial arteries. J Anat 1995; 187: 473-79.

22. Rodriguez-Niedenfuhr M, Sannudo JR, Vazquez T, Nearn L, Logan B, Parkin I. Median artery revisited. J Anat 1999; 195: 57-63.

23. Rodriguez-Niedenfuhr M, Sannudo JR, Vazquez T, Nearn L, Logan B, Parkin I. Anastomosis at the level of the elbow joint connecting the deep, or normal, brachial artery with major arterial variations of the upper limb. J Anat 2000; 196 $115-9$.

24. Saeed M, Rufai AA. Median and musculocutaneous nerves: variant formation and distribution. Clin. Anat 2003; 16(5): 453-7.

25. Taylor GI, Cichowitz A, Ang SG, Seneviratne S, Ashton M. Comparative anatomical study of the gracilis and coracobrachialis muscle: implications for facial reanimation. Plast Reconstr Surg 2003; 112(1): 20-30.

26. Uzun A, Seeling LL. A variation in the formation of the median nerve: communicating branch between the musculocutaneous and median nerves in man. Folia Morphol (Warsz) 2001; 60(2): 99-101.

27. Wadhwa S, Vasudeva N, Kaul JM. Rare constellation of multiple upper limb anomalies. Folia Morphol (Warsz) 2008; 67(4): 236-9.

Received: 25/06/2012

Accepted in revised form: 11/11/2012

\section{Corresponding author:}

Dr. Surbhi Wadhwa, C1/1, All India Institute of Medical Sciences, Ansari Nagar, New Delhi-110029, India, e-mail: wadhwa.surbhi@gmail.com 